\title{
Study of the denoising and feature extrac- tion based on eyes-closed EEG signal
}

\author{
Nianqiang Li Yongling Wang \\ Shandong Provincial Key Laboratory of Network Based Intelligent Computing; \\ School of Information Science and Engineering; University of Jinan
}

\begin{abstract}
The electroencephalogram (EEG) is a kind of biological signal that represents the activity of the brain which plays a key role in the diagnosis of brain. EEG signal was de-noised using butterworth filter in this paper. Based on analysis of methods of EEG signal processing, we propose to use wavelet transform combined with ICA for EEG feature extraction. The noise is successfully removed and the feature wave $\alpha$ of eyes-closed EEG signal is successfully extracted with the new mothed.
\end{abstract}

Keywords: Butterworth filter; wavelet transform; feature extraction; ICA

\section{Introduction}

EEG signal as a stationary signal including a lot of important information but also a lot of noise. How to process and analyze EEG is significant. Butterworth filter is an important tool in EEG denoising. In this paper combing the Butterworth filter with wavelet transform and ICA is proved that the effect is good. Wavelet transform method ${ }^{[1]}$ which is based on multi- resolution analysis is introduced in this thesis. First, the notion of wavelet transform theory is illustrated succinctly at the beginning of this thesis. Second, we apply the method to decompose EEG signal into seven layers and reconstruct each layer of the signal. Wavelet transform method can weaken interference composition, and then we enhance the useful information by $\mathrm{ICA}^{[2]}$, the algorithm we use is fast ICA algorithm. The paper shows a new method to enhance the transient characteristics with the ICA algorithm ${ }^{[3]}$. With the method combining ICA and wavelet transform for feature extraction and denoising with butterworth filter, we extract $\alpha$ wave successfully.

\section{Materials and method}

\subsection{Data collection}

The data set comes from our laboratory. The experimenter was required to close his eyes with thinking nothing for several minutes. EEG data was digitally recorded at a rate of 100 samples/s using EEG acquisition equipment, (High frequency filter is $30 \mathrm{~Hz}$. Time constant is $0.3 \mathrm{~s}$ ).

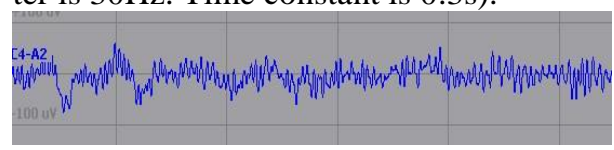

Fig.1: Eyes closed EEG signal of C4 channel.

Electrodes were placed according to the 16-19 system, at the following 16 locations: Fp1, F3, C3, P3, O1, F7, T3, T5, Fp2, F4, C4, P4, O2, F8, T4 and T6 with a reference at Fcz. We chose 2 to $3 \mathrm{mi}-$ nute, a time at which the slow waves are the most prominent. In view of more easier to deal with data, we selected one channel to analysis data when the experimenter eyes open and closed. 


\subsection{Butterworth filter}

Butterworth filter is also called BWLP filter. Its norm of amplitude response is defined as bellow:

$$
|H(\mathrm{j} \omega)|^{2}=\frac{1}{1+\left(\omega / \omega_{c}\right)^{2 N}}
$$

Generally, $\omega_{\mathrm{c}}$ is calculated as the formula bellow:

$$
\frac{\omega_{\mathrm{p}}}{\left(10^{0.1 A_{p}}-1\right)^{1 / 2 n}} \leq \omega_{c} \leq \frac{\omega_{s}}{\left(10^{0.1 A_{p}}-1\right)^{1 / 2 N}}
$$

In this paper, the parameters $\omega_{\mathrm{p}}$ is 0.25 $\pi \mathrm{rad} / \mathrm{s}, \omega_{\mathrm{s}}$ is $0.3 \pi \mathrm{rad} / \mathrm{s}$.

\subsection{Wavelet transform}

The wavelet transform is designed to address the problem of non-stationary signals. EEG signal is a non-stationary signal. The definition of wavelet transform is

$$
C W T(a, b)=\int_{-\infty}^{+\infty} x(t) \Psi_{a, b}^{*}(t) d t
$$

$\Psi_{a, b}(t)$ which is obtained by scaling the wavelet at time $b$ and scale $a$ :

$$
\Psi_{a, b}(t)=\frac{1}{\sqrt{|a|}} \Psi\left(\frac{t-b}{a}\right)
$$

where ${ }^{\Psi(t)}$ represents the wavelet ${ }^{[4]}$.

Because of calculating wavelet coefficients for every possible scale can represent a considerable effort and result in a vast amount of data, therefore discrete wavelet transform (DWT) is often used.

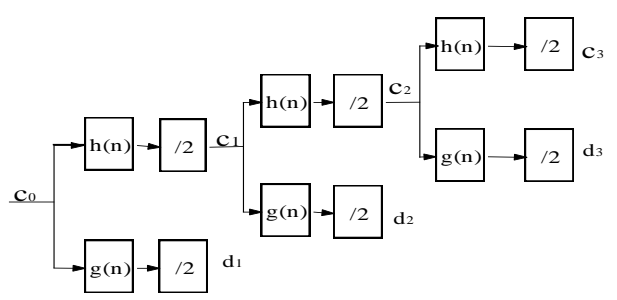

Fig.2: Sub-band decomposition of discrete wavelet transform implementation.

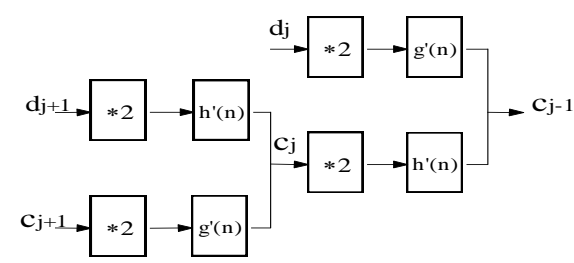

Fig.3: Sub-band reconstruction of discrete wavelet transform implementation.

We decomposed the EEG signal into 7 layers with mallet algorithm. We chose the frequency band we need as the feature.

\subsection{ICA algorithm}

With negative entropy maximum as a search for direction, Fast ICA algorithm adopts Fixed-point iterative optimization algorithm, Fast ICA algorithm iterative formula is,

$$
\begin{aligned}
& W_{i}(k+1)=E\left[z f\left(W_{i}^{T}(k) z\right)\right]-E\left[f^{\prime}\left(W_{i}^{T}(k) z\right)\right] W_{i}(k) \\
& W_{i}(k+1) \leftarrow \frac{W_{i}(k+1)}{\left\|W_{i}(k+1)\right\|_{2}}
\end{aligned}
$$

Using ICA method ${ }^{[5]}$ after first feature extraction to enhance the feature information, we extract the alpha rhythm completely.

\subsection{EEG denoising}

First we create Butterworth filter with the design index. The Butterworth we designed as showed in Figure. 4.

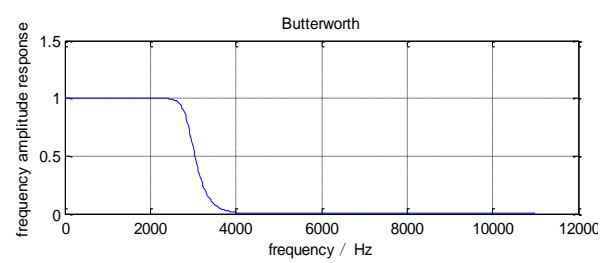

Fig.4: Butterworth filter we designed.

Filter application to the original EEG signal is showed in the Figure.5. Comparing with the power spectrum in the Figure. 5 before filter, we can see the high frequency of the EEG is filtered. 

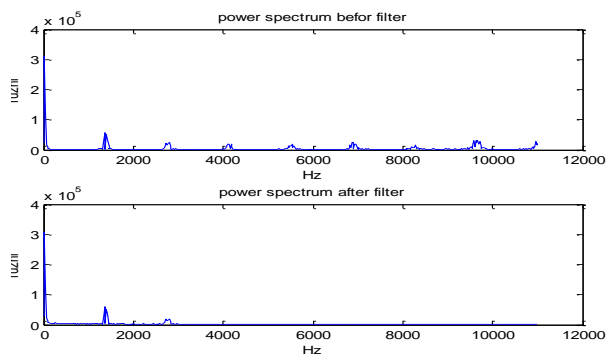

Fig.5: Power spectrum before filtering and after filtering.

\subsection{Applying wavelet transform to EEG data}

We selected the F3 channel from both eyes open and closed EEG data. Considering the accuracy and the frequency of the alpha wave, we decomposed the EEG signal into 7 layers by using DB4 wavelet ${ }^{[6]}$. The sampling frequency of EEG signal is $1000 \mathrm{HZ}$. According to the formula,

$\left[0, \frac{f_{s}}{2}\right]=\left[0, \frac{f_{s}}{2^{L+1}}\right] \cup\left[\frac{f_{s}}{2^{L+1}}, \frac{f_{s}}{2^{L}}\right] \cup \ldots \cup\left[\frac{f_{s}}{2^{2}}, \frac{f_{s}}{2}\right]$

The alpha wave frequency is between $8 \mathrm{~Hz}$ and $13 \mathrm{~Hz}$. As shown in the figure, we can see decomposition structure contains the coefficients of wavelet decomposition $\mathrm{C}$ and coefficient vector $\mathrm{L}$ showed in Figure.6.
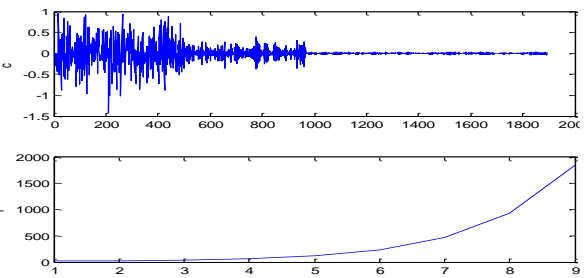

Fig.6:Decomposition vector $\mathrm{C}$ and coefficient length of eyes closed.

The extracted wavelet coefficients provide a compact representation that shows the energy distribution of the EEG signal in time and frequency. The Figure. 5 presents the frequencies corresponding to different levels of decomposition for DB4 wavelet with a sampling frequency of $100 \mathrm{~Hz}$.According to the reconstruction of wavelet equation, we can also get the reconstruction coefficients of each subband .

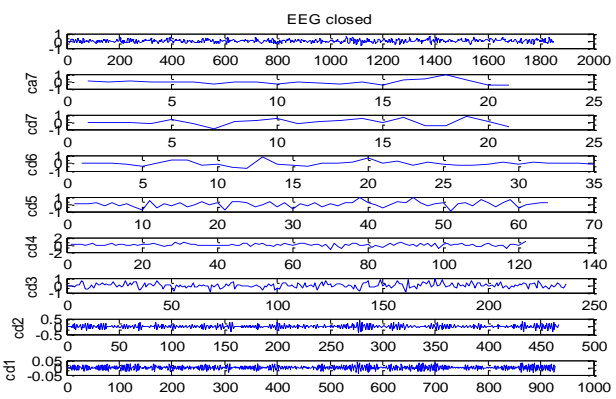

Fig.7: Decomposition coefficients in each sub-band.

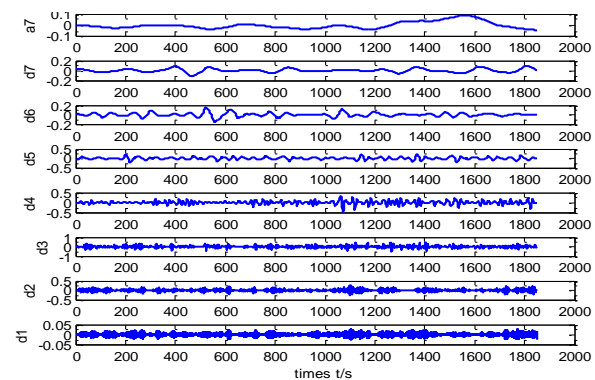

Fig.8: Reconstruction coefficients in each sub-band.

Then we did FFT to the eyes closed EEG data. According to the frequency band, we know the alpha wave is $8 \sim 13 \mathrm{~Hz}$, therefore we can get the wave band we need. The alpha rhythm is in the D5 layer. The Figure.9 presents the extraction wave and time frequency distribution of the EEG signals.
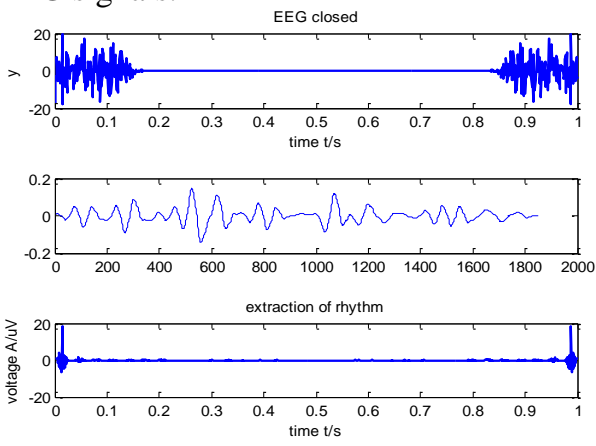

Fig.9: Alpha rhythm and time frequency distribution. 
We did FFT again to the new data. After calculating the corresponding amplitude of different points, we concluded its power spectrum and the corresponding frequency value of each point.

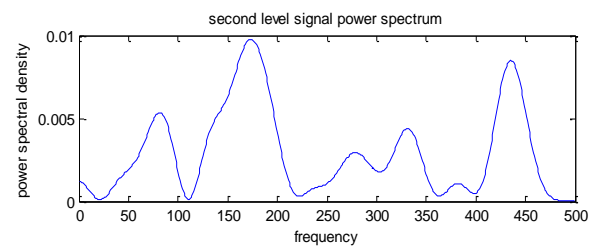

Fig.10: Signal power spectrum of eyes closed.

At the same time, we did the same procedure on the data when the experimenter eyes open. Its corresponding frequency value of each point is shown as bellow:

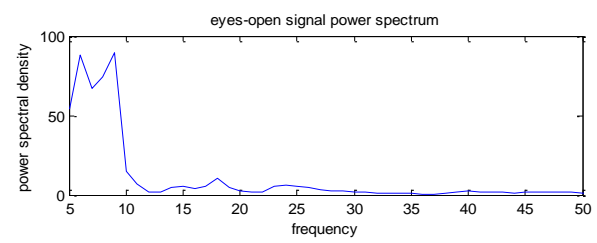

Fig.11: Signal power spectrum of eyes open.

Comparing the Figure.11, Figure.12 shows the power spectrum while eyes closed, thus it follows that alpha rhythm is more obvious when the experimenter eyes closed.

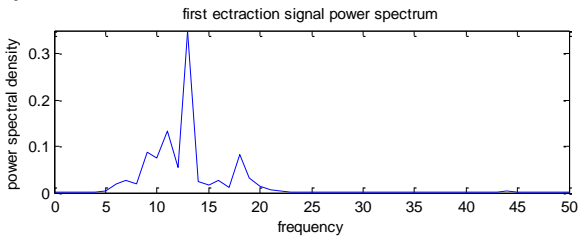

Fig.12: Signal power spectrum of eyes closed.

\section{Feature enhancing with ICA}

We process the sub-band EEG signal restructuring using Fast ICA algorithm. Comparing the results after ICA processing, we can see the $\alpha$ wave is enhanced and better than the result without ICA processing .

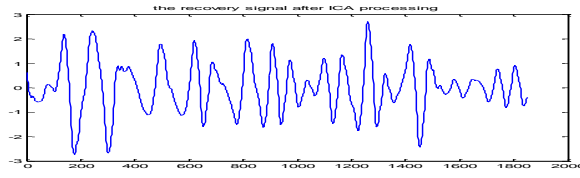

Fig.13: $\alpha$ wave extracted using new method with eyes closed.

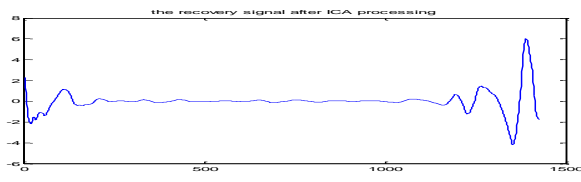

Fig.14: $\alpha$ wave extracted using new method with eyes opened.

\section{The results of simulation analysis}

We simulated by MATLAB 7.1.The method with filter by combining wavelet transform and ICA comparing with methods including wavelet transform with or without filter, ICA with or without filter, and the two combined without filter. The results are as follows:
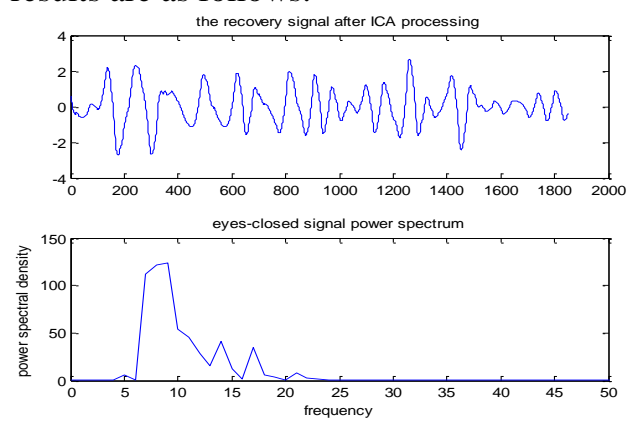

Fig.15: $\alpha$ wave extracted by new method and power spectrum.
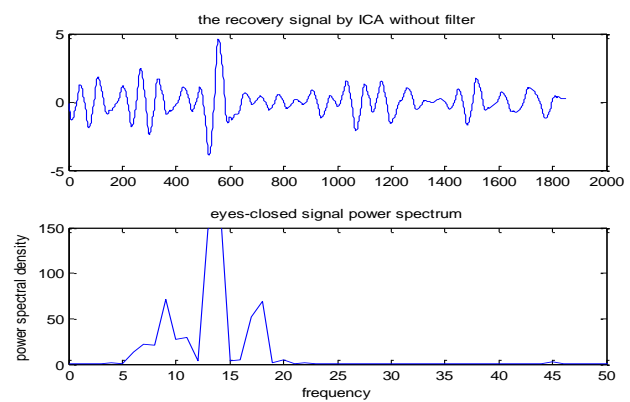

Fig.16: $\alpha$ wave extracted by ICA without filter. 

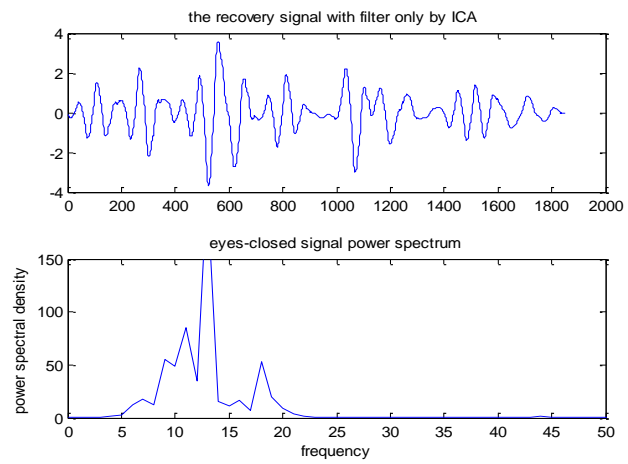

Fig. 17: $\alpha$ wave extracted by ICA with filter.
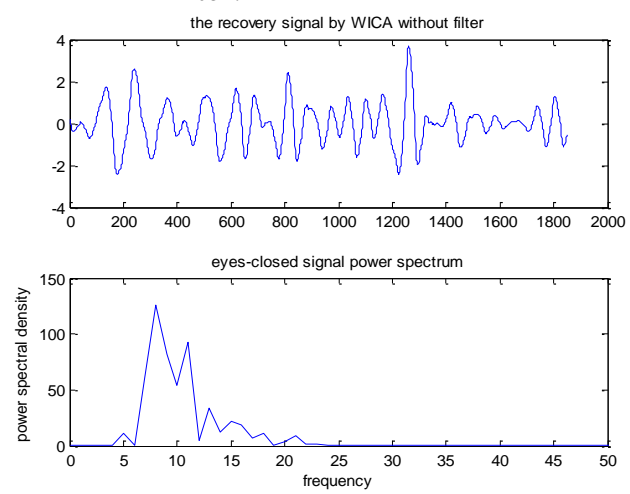

Fig.18: the $\alpha$ wave extracted by ICA and wavelet transform without filter.

Comparing the power spectrum of each method, because of the frequency of $\alpha$ wave band is $8-12 \mathrm{HZ}$, we can conclude that the result of the new method in this paper is better than other method. The results of the experiment show the new method is effective.

\section{Conclusion}

The basic requirement to find an accurate model is the collection of well distributed, sufficiently, and accurately measured input data. This study tells us that every rhythm has a clear physical meaning. Butterworth filter can effectively filter the noise. Wavelet transform can enhance the composition of the signal to be detected, weaken the off-target signal composition and noise, at the same time, ICA algorithm can effectively isolated relatively strong target signal components. We can capture the important information from this method introduced in this article which is also a significant part in the signal feature extraction.

\section{References}

[1] W. Jing, "Based on wavelet decomposition of the imagination about the feature extraction of EEG classification," East China Jiao tong University, 2008.

[2] W. d. Zhou, and J. Gotman, "Automatic removal of eye movement artifacts from the EEG using ICA and the dipole model," Progress in Natural Science, pp. 1165-1170, 2009.

[3] L. Hu, Z.G. Zhang, Y.S. Hung, K.D.K. Luk, G.D. Iannetti, and Y.Hu, "Single-trial detection of somatosensory evoked potentials by probabilistic independent component analysis and wavelet filtering," Clinical Neurophysiology, pp. 1429-1423, 2011.

[4] E. V. Orekhova, M. Elam, and V. Y.. Orekhov, "Unraveling superimposed EEG rhythms with multidimensional decomposition," Journal of Neuroscience Methods, pp. 4760, 2011.

[5] Maarten D V, Lieven D L and Sabine $\mathrm{V} \mathrm{H}$, "Spatially constrained ICA algorithm with an application in EEG processing J," Signal Processing, pp. 1963-72, 2011.

[6] W. Y. Hsu, C. H. Lin, H. J. Hsu, P. H. Chen, and I. R. Chen, "Wavelet-based envelope features with automatic EOG artifact removal: Application to single-trial EEG data," Expert Systems with applications, pp. 27432749,2012 . 